\title{
Versatile low-Reynolds-number swimmer with three-dimensional maneuverability
}

\author{
Mir Abbas Jalali ${ }^{1,2}$ - Mohammad-Reza Alam ${ }^{3}{ }^{\dagger}$ and SeyyedHossein Mousavi ${ }^{2}$ \\ ${ }^{1}$ Department of Astronomy, University of California, Berkeley, CA 94720, USA \\ ${ }^{2}$ Department of Mechanical Engineering, Sharif University of Technology, Azadi Avenue, Tehran, Iran \\ ${ }^{3}$ Department of Mechanical Engineering, University of California, Berkeley, California 94720, USA
}

(Dated: July 18, 2021)

\begin{abstract}
We design and simulate the motion of a new swimmer, the Quadroar, with three dimensional translation and reorientation capabilities in low Reynolds number conditions. The Quadroar is composed of an I-shaped frame whose body link is a simple linear actuator, and four disks that can rotate about the axes of flange links. The time symmetry is broken by a combination of disk rotations and the one-dimensional expansion/contraction of the body link. The Quadroar propels on forward and transverse straight lines and performs full three dimensional reorientation maneuvers, which enable it to swim along arbitrary trajectories. We find continuous operation modes that propel the swimmer on planar and three dimensional periodic and quasi-periodic orbits. Precessing quasi-periodic orbits consist of slow lingering phases with cardioid or multiloop turns followed by directional propulsive phases. Quasi-periodic orbits allow the swimmer to access large parts of its neighboring space without using complex control strategies. We also discuss the feasibility of fabricating a nano-scale Quadroar by photoactive molecular rotors.
\end{abstract}

PACS numbers: 87.19.ru,47.15.G-,45.40.Ln

\section{INTRODUCTION}

The dynamics of a self-propelling object in a viscous fluid is characterized by the Reynolds number that determines the relative importance of inertia forces against viscous ones. When the Reynolds number is small, the exchange of momentum between the object and the background fluid is small and the object is driven mainly by drag forces. The frictional interaction between the object and the background fluid is only a necessary condition for swimming at low Reynolds numbers, for the Stokes equation governing the flow of the background fluid remains invariant under the reflection transformations $t \rightarrow-t$ over the time domain, and consequently, a swimmer cannot advance in the configuration space through reciprocal movements or deformations of its body. This is why swimmers consisting of a finite number of rigid components need at least two degrees of freedom to break the time symmetry and stroke [1, 2].

Artificial low-Reynolds-number swimmers have applications in both the micro and macro scales. In micronscales, they can deliver drug and cargo to cells, and interact with micro-organisms and bacteria. In large macroscales they can conduct inspection missions in oil tanks and highly viscous reservoirs. Like any robot, and regardless of the mechanism and swimming strategy, the major technological challenge of building swimmers in low Reynolds number conditions is how to power their actuators and control them onboard or remotely. Therefore, not all proposed swimmers can be engineered and operated in reality. The simplest low-Reynolds-number swimmer is the linked three-sphere of Najafi and Golestanian

\footnotetext{
* mjalali@berkeley.edu

$\dagger$ reza.alam@berkeley.edu
}

3] that rectilinearly swims using two telescopic actuators. The hydrodynamics, global controllability and optimal stroking of the Najafi-Golestanian swimmer have been studied in detail [4 [6] and experiments with an optical tweezer have shown that the three-sphere system can indeed generate a net flow [7], necessary for swimming. The rotating version of this swimmer has been proposed in [8]. Pushme-pullyou is another promising rectilinear swimmer 9] that uses one telescopic actuator and two spherical bladders that exchange their volume in each stroke.

Low-Reynolds-number swimming in two or three dimensions can be performed using triangular or tetrahedral configurations of linked spheres [10, 11] and twosphere propellers [12]. The main advantage of the circle swimmer proposed in [10] is that it can move on complex planar trajectories only by controlling the angle between its two links. Deformable bodies [13 15$]$ and articulated robots with rigid links [16] have also been suggested for low-Reynolds-number swimming. Nonetheless, the realization of swimmers with deformable surfaces and with intuitive bladders as in pushme-pullyou [9] is a technological challenge, especially for two- and three-dimensional swims. Moreover, swimmers with many linked spheres and articulated $N$-link tails are not optimum systems because of two reasons: they require a complex control strategy and too many degrees of freedom to perform a planned maneuver, and they experience lateral drifts in each stroke by generating unnecessary flows perpendicular to the motion direction.

In this study, we propose the concept and analyze the motion of a swimmer with five degrees of freedom that combines the rotations of four disks (oars) and the telescopic motion of its body link, so follows the name Quadroar $=$ Quadru+oar, to perform full three dimensional movements. The swimmer has three main features: 
(i) It can perform reorientation maneuvers by rotating about all of its three principal body axes, and stroke along prescribed three dimensional curves without unnecessary side drifts. (ii) Its concept can be realized from nano to macro scales, with a variety of scientifically feasible and technologically available actuation mechanisms. (iii) Similar to what the circle swimmer [10] can perform in two dimensions, it can track prescribed 3D paths using stepwise control strategies, or move on quasi-periodic orbits by the continuous operation of its actuators. Quasiperiodic orbits become dense in certain regions of the configuration space, and enable the swimmer to access large parts of its neighboring space.

The paper is organized as follows. We introduce the geometry and degrees of freedom of the Quadroar in section $\Pi$ and derive its equations of motion in section III where we also discuss its independent operation modes that lead to directional motions and rigid body maneuvers by pulsed commands to its actuators. Section IV is dedicated to swimming modes with continuous operations of main actuators. We also explore the parameter space to generate a wide range of quasi-periodic orbits in two and three dimensions. Section $\square$ concludes the paper by discussing the unique features of the Quadroar, its possible applications, and its realization in nano scales.

\section{GEOMETRY AND KINEMATICS}

Figure 1 shows the geometry of Quadroar that consists of three links with an I-shape arrangement and four similar disks of radius $a$. The length of the body link continuously varies by a linear actuator, and disks can rotate continuously or stepwise about the axes of flange links. From here on, we refer to the set of the links as the "I-frame". The length of the body link is $2 l+2 s$ where $2 s$ is the contribution from the expansion/contraction of the linear actuator. The front and rear flange links have constant lengths of $2(b-a)$ (the distance from the cross section of the body link and a flange link to the center of each disk is $b$ ). The rotation of the $n$-th disk ( $n=1,2,3,4)$ about its supporting flange link is measured by the angle $-\pi \leq \vartheta_{n} \leq \pi$ that the plane of the disk makes with the I-frame.

We denote the inertial coordinate system and its corresponding basis vectors by $\left(X_{1}, X_{2}, X_{3}\right)$ and $\left(\boldsymbol{E}_{1}, \boldsymbol{E}_{2}, \boldsymbol{E}_{3}\right)$, respectively. The translational motion of the swimmer is thus described by the position of its center of mass, $\boldsymbol{X}_{\mathrm{c}}=\left(X_{1 \mathrm{c}}, X_{2 \mathrm{c}}, X_{3 \mathrm{c}}\right)$, and its orientation is determined by the roll-pitch-yaw angles $\boldsymbol{\alpha}=(\phi, \theta, \psi)$. Consider a body-fixed coordinate frame $\left(x_{1}, x_{2}, x_{3}\right)$, with the unit vectors $\left(\boldsymbol{e}_{1}, \boldsymbol{e}_{2}, \boldsymbol{e}_{3}\right)$, whose $x_{1}$-axis is along the body link and $x_{3}$-axis is perpendicular to the I-frame (Fig. 1). Defining $\mathbf{R}_{x_{i}}(\gamma)$ as a rotation matrix about the $x_{i}$-axis by the angle $\gamma$, transformation from $\boldsymbol{E}_{i}$ to $\boldsymbol{e}_{i}(i=1,2,3)$ is carried out using the successive operation of the matrices $\mathbf{R}_{x_{3}}(\psi), \mathbf{R}_{x_{2}}(\theta)$ and $\mathbf{R}_{x_{1}}(\phi)$ (see Appendix $\underline{\mathbf{A}}$ ), and
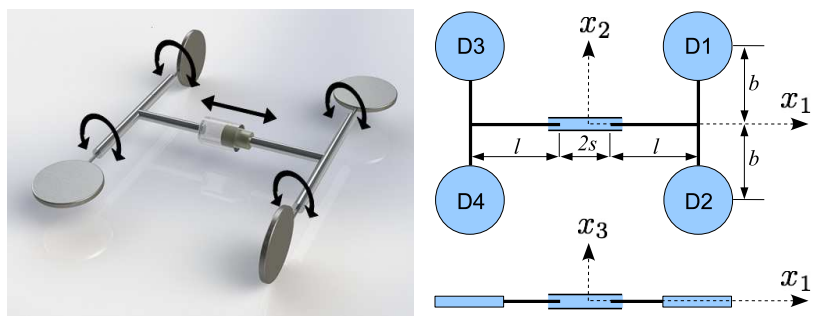

FIG. 1. The geometry of the Quadroar. The body link of the I-frame is a linear actuator, here schematically shown as a telescopic component. Four disks are mounted at the end points of flange links. Each disk has a single rotational degree of freedom about the axis of its supporting link. The bodyfixed coordinate frame is such that the I-frame lies in the $\left(x_{1}, x_{2}\right)$-plane.

we obtain

$$
\boldsymbol{E}_{i}=\mathbf{R} \cdot \boldsymbol{e}_{i}, \quad \mathbf{R} \equiv R_{i j} \boldsymbol{e}_{i} \boldsymbol{e}_{j}=\mathbf{R}_{x_{1}} \cdot \mathbf{R}_{x_{2}} \cdot \mathbf{R}_{x_{3}} .
$$

Here and throughout the paper, repeated indices associated with the components of vectors or elements of tensors denote summations over those indices. Assuming that the linear actuation of the body link does not change the location of swimmer's center of mass, and denoting the angular velocity of the swimmer in the body frame by $\Omega$, the velocity of the $n$th disk measured at its center of mass is obtained from

$$
\begin{aligned}
& \boldsymbol{v}_{n}=\mathbf{R} \cdot \boldsymbol{v}_{\mathrm{c}}+\dot{\ell}_{n} \boldsymbol{e}_{1}+\boldsymbol{\Omega} \times \boldsymbol{r}_{n}, \quad \boldsymbol{v}_{\mathrm{c}}=\dot{\boldsymbol{X}}_{\mathrm{c}}, \\
& \boldsymbol{r}_{n}=r_{n, i} \boldsymbol{e}_{i}=\ell_{n} \boldsymbol{e}_{1}+b_{n} \boldsymbol{e}_{2},
\end{aligned}
$$

where the over-dot denotes $d / d t$ and

$$
\begin{aligned}
& \ell_{n}=\left\{\begin{array}{l}
+(l+s) \text { for } n=1,2 \\
-(l+s) \text { for } n=3,4
\end{array},\right. \\
& b_{n}=\left\{\begin{array}{l}
+b \text { for } n=1,3 \\
-b \text { for } n=2,4
\end{array},\right.
\end{aligned}
$$

The linear actuator expands when $\dot{s}>0$ and contracts when $\dot{s}<0$. For the sake of compact notation, let $s_{\gamma}$ and $c_{\gamma}$ denote $\sin (\gamma)$ and $\cos (\gamma)$, respectively. Since the disks can rotate with respect to the frame, their absolute angular velocities become

$$
\omega_{n}=\boldsymbol{\Omega}+\dot{\vartheta}_{n} \boldsymbol{e}_{2}
$$

where

$$
\boldsymbol{\Omega}=\mathbf{T} \cdot \dot{\boldsymbol{\alpha}}, \quad \mathbf{T}=\left[\begin{array}{ccc}
1 & 0 & -s_{\theta} \\
0 & c_{\phi} & c_{\theta} s_{\phi} \\
0 & -s_{\phi} & c_{\theta} c_{\phi}
\end{array}\right] .
$$

The transformation matrix $\mathbf{T}$ has a coordinate type singularity (gimbal lock) at $\theta= \pm \pi / 2$. This singularity is avoided by carrying out attitude computations in the space of unit quaternions. Dynamics of quaternions and their relationship with roll-pitch-yaw angles are discussed in Appendix B. With five control inputs, $s(t)$ and $\vartheta_{n}(t)$, the Quadroar is an under-actuated system that can move 
along, and rotate about, any of its body axes except performing side slip along the $x_{2}$ axis. In section [II] we will show that being under-actuated does not restrict the three-dimensional maneuvering and tracking capability of the Quadroar.

\section{SWIMMING AND REORIENTATION}

We assume that the effective cross section (area) of the I-frame is negligible compared to four disks, and therefore, the drag forces and torques that the frame links experience are ignored. The needed driving force for translation and the torque for rigid-body rotation come from the disks, which feel fluid resistance as they move and rotate. We also work with a mechanism that preserves the location of the swimmer's center of mass during the actuation of the body link. One more simplifying assumption is that the disks are apart enough so that the fluid streaming around each disk is not affected by the movement of the frame or other disks. This requirement confines us to models with $a \ll l, b$.

For a background fluid of dynamic viscosity $\mu$, and in the absence of accelerations, the translation tensor of a circular disk is given by equation (5-4.29) in [17]:

$$
\mathbf{K}=\left(\frac{32}{3} \boldsymbol{i}_{1} \boldsymbol{i}_{1}+\frac{32}{3} \boldsymbol{i}_{2} \boldsymbol{i}_{2}+16 \boldsymbol{i}_{3} \boldsymbol{i}_{3}\right) a
$$

where $\left(\boldsymbol{i}_{1}, \boldsymbol{i}_{2}, \boldsymbol{i}_{3}\right)$ define an orthogonal coordinate system fixed to the disk. The origin of the coordinate system coincides with the center of mass of the disk and $\boldsymbol{i}_{3}$ is normal to the disk plane. The drag force that the $n$th disk experiences as it moves with the velocity $\boldsymbol{v}_{n}$ thus reads

$$
\boldsymbol{F}_{n}=-\mu \mathbf{K} \cdot \boldsymbol{v}_{n}
$$

When the $n$th disk is actuated, its axis of rotation always remains along the $x_{2}$-axis of the I-frame. It is therefore a computational convenience to set $\boldsymbol{i}_{2}=\boldsymbol{e}_{2}$ and obtain

$$
\left(\begin{array}{c}
\boldsymbol{i}_{1} \\
\boldsymbol{i}_{2} \\
\boldsymbol{i}_{3}
\end{array}\right)=\left[\begin{array}{ccc}
\cos \left(\vartheta_{n}\right) & 0 & \sin \left(\vartheta_{n}\right) \\
0 & 1 & 0 \\
-\sin \left(\vartheta_{n}\right) & 0 & \cos \left(\vartheta_{n}\right)
\end{array}\right] \cdot\left(\begin{array}{l}
\boldsymbol{e}_{1} \\
\boldsymbol{e}_{2} \\
\boldsymbol{e}_{3}
\end{array}\right)
$$

We note that the rotation angle $\vartheta_{n}$ is positive if its angular velocity vector is along the positive $x_{2}$-axis. Substitution of (10) into (8) results in the translation tensor $\mathbf{K}_{n}=K_{n, i j} \boldsymbol{e}_{i} \boldsymbol{e}_{j}$ of the $n$th disk with respect to the frame of the swimmer:

$$
\mathbf{K}_{n}=\frac{8}{3} a\left[\begin{array}{ccc}
5-\cos \left(2 \vartheta_{n}\right) & 0 & \sin \left(2 \vartheta_{n}\right) \\
0 & 4 & 0 \\
\sin \left(2 \vartheta_{n}\right) & 0 & 5+\cos \left(2 \vartheta_{n}\right)
\end{array}\right]
$$

In the low-Reynolds regime the acceleration of the swimmer is small. Therefore, if the swimmer is designed to be neutrally buoyant, the sum of the drag forces
$\boldsymbol{F}_{n}=-\mu \mathbf{K}_{n} \cdot \boldsymbol{v}_{n}$ vanishes and we obtain the force balance equation

$$
\mu \sum_{n=1}^{4} \mathbf{K}_{n} \cdot \boldsymbol{v}_{n}=0
$$

The resistive torque that a circular disk experiences due to its pure rotation is calculated from [17]

$$
\boldsymbol{\tau}_{n}=-\mu \mathbf{G} \cdot \boldsymbol{\omega}_{\boldsymbol{n}}, \quad \mathbf{G}=\frac{32}{3} a^{3} \mathbf{I}
$$

where $\mathbf{I}$ is the identity matrix and $\mathbf{G}$ is the rotation tensor, which is isotropic at the center of the disk. The resultant torque exerted on the swimmer is the superposition of $\boldsymbol{\tau}_{n}$ with the torques $\boldsymbol{r}_{n} \times \boldsymbol{F}_{n}$ that the drag forces generate about the center of mass of the swimmer. Consequently, the torque balance equation becomes

$$
\mu \sum_{n=1}^{4}\left[\mathbf{G} \cdot \boldsymbol{\omega}_{n}+\boldsymbol{r}_{n} \times\left(\mathbf{K}_{n} \cdot \boldsymbol{v}_{n}\right)\right]=0 .
$$

On substituting from equations (2) and (6) into the force and torque balance equations, and after some tensorial manipulations to separate $\boldsymbol{v}_{\mathrm{c}}$ and $\boldsymbol{\Omega}$, we obtain

$$
\left[\begin{array}{ll}
\mathbf{C}_{11} & \mathbf{C}_{12} \\
\mathbf{C}_{21} & \mathbf{C}_{22}
\end{array}\right] \cdot\left(\begin{array}{c}
\boldsymbol{v}_{\mathrm{c}} \\
\boldsymbol{\Omega}
\end{array}\right)=\left(\begin{array}{l}
f_{1} \\
f_{2}
\end{array}\right)
$$

which is a more convenient form for motion analysis and control. The elements of the resistance matrices $\mathbf{C}_{\alpha \beta}$ and the components of the forcing vectors $\boldsymbol{f}_{\alpha}(\alpha, \beta=1,2)$ have been given in Appendix A. Equations (15) constitute a system of nonlinear ordinary differential equations for the roll-pitch-yaw angles with parametric and external excitations through the matrices $\mathbf{K}_{n}$ and vectors $\boldsymbol{f}_{\alpha}$. Once $\boldsymbol{\alpha}(t)$ is computed in terms of quaternions (see Appendix (B), the position of the swimmer is found by integrating $\dot{\boldsymbol{X}}_{\mathrm{c}}=\boldsymbol{v}_{c}$.

Quadroar's movements are initialized by setting $s=0$ for the body link and putting the disks in appropriate park configurations. This is done in a way that the net drag-induced force and torque remain zero while adjusting the disks. Let us define the input control signal of the variable $u$ by a simple pulse function in its phase space: $\dot{u}=\nu_{u}(t)$ for $u_{\mathrm{i}} \leq u \leq u_{\mathrm{f}}$ and $\dot{u}=0$ otherwise. Here $u_{\mathrm{i}}$ and $u_{\mathrm{f}}$ are the initial and terminal conditions of $u$, and $\nu_{u}$ is a linear or angular velocity. We simply define $\dot{\vartheta}_{n}=\nu_{n}(t)$ for disks. One may collect the three variables $u_{\mathrm{i}}, \nu_{u}$ and $u_{\mathrm{f}}$ of each actuator in a single vector and define the control signals

$$
\mathcal{S}=\left(\begin{array}{c}
s_{\mathrm{i}} \\
\nu_{s} \\
s_{\mathrm{f}}
\end{array}\right), \quad \mathcal{D}=\left[\begin{array}{c|c|c|c}
\vartheta_{1 \mathrm{i}} & \vartheta_{2 \mathrm{i}} & \vartheta_{3 \mathrm{i}} & \vartheta_{4 \mathrm{i}} \\
\nu_{1} & \nu_{2} & \nu_{3} & \nu_{4} \\
\vartheta_{1 \mathrm{f}} & \vartheta_{2 \mathrm{f}} & \vartheta_{3 \mathrm{f}} & \vartheta_{4 \mathrm{f}}
\end{array}\right] .
$$

Two signals that differ by the signs of $\nu_{u}$ and have interchanged their initial and final states will be denoted by \pm superscripts. For instance, $\mathcal{D}^{-}$is obtained from $\mathcal{D}^{+}$ if the elements in the second row of $\mathcal{D}^{+}$flip sign, and 


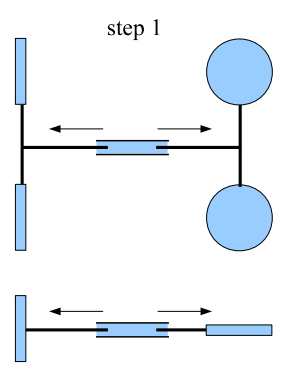

(a)
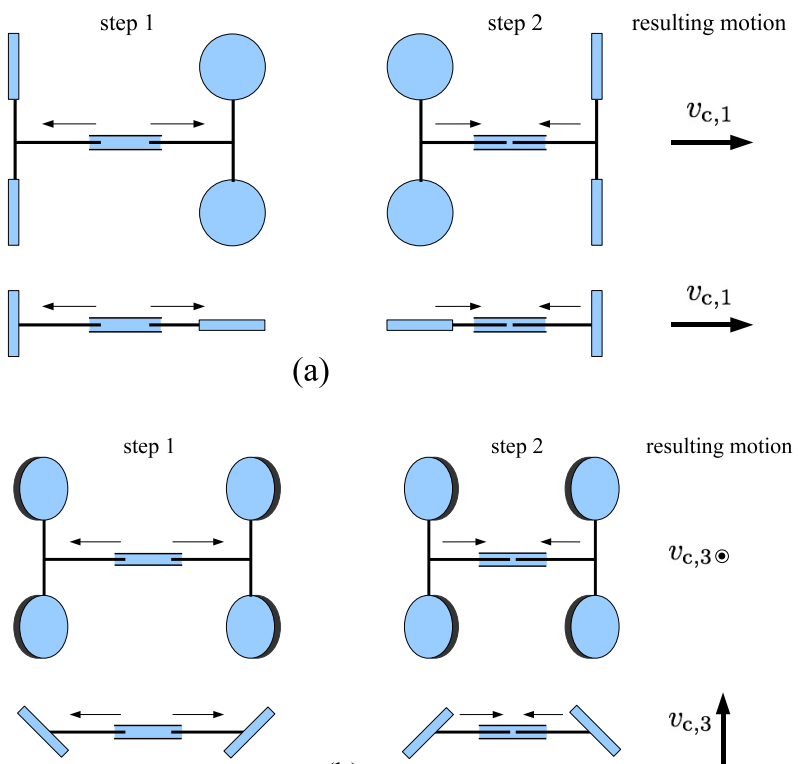

(b)

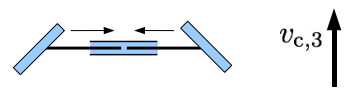

FIG. 2. (a) Direct strokes in the $x_{1}$-direction using a pushmepullyou type sequence. (b) Transverse rigid-body translation in the direction normal to the plane of the I-frame. In both swimming modes, disks rotate and acquire their desired orientations before the expansion or contraction of the body link begins. The simultaneous rotations of the disks before each step are such that the torque corresponding to the first (isotropic) term in equation (14) vanishes and we get $\boldsymbol{\Omega}=\mathbf{0}$.

the first and thirds rows are interchanged. The simplest swimming/rotation modes are obtained when spatial displacements and rigid-body rotations are decoupled. Below, we further explore such operation modes.

The first swimming mode begins with the initializing signal

$$
\mathcal{D}_{0}=\left[\begin{array}{c|c|c|c}
0 & 0 & 0 & 0 \\
0 & 0 & \nu_{3} & -\nu_{3} \\
0 & 0 & \frac{\pi}{2} & -\frac{\pi}{2}
\end{array}\right],
$$

that puts D3 and D4 in a vertical position (with respect to the I-frame as shown in the step 1 of Fig. 2(a)) while keeping $\boldsymbol{v}_{\mathrm{c}}$ and $\boldsymbol{\Omega}$ equal to zero. We then lock the disks and command the body link to expand with $\nu_{s}>0$, which sets $\mathcal{S}^{+}$. This keeps $\boldsymbol{\Omega}=\mathbf{0}$ and generates a stroke in the $x_{1}$-direction. To compute the advancing velocity $v_{\mathrm{c}, 1}$, we write the force balance equation in the form

$$
\begin{aligned}
\left(\mathbf{K}_{1}\right. & \left.+\mathbf{K}_{2}\right) \cdot\left(\boldsymbol{v}_{\mathrm{c}}+\nu_{s} \boldsymbol{e}_{1}\right) \\
& +\left(\mathbf{K}_{3}+\mathbf{K}_{4}\right) \cdot\left(\boldsymbol{v}_{\mathrm{c}}-\nu_{s} \boldsymbol{e}_{1}\right)=0,
\end{aligned}
$$

where

$$
\begin{aligned}
& \mathbf{K}_{1}=\mathbf{K}_{2}=\frac{8}{3} a \cdot \operatorname{diag}[5-1,4,5+1], \\
& \mathbf{K}_{3}=\mathbf{K}_{4}=\frac{8}{3} a \cdot \operatorname{diag}[5+1,4,5-1] .
\end{aligned}
$$

Equation (18) then gives $v_{\mathrm{c}, 1}=\nu_{s} / 5, v_{\mathrm{c}, 2}=0$ and $v_{\mathrm{c}, 3}=$ 0 . Stopping the expansion of the body link at $s=s_{\mathrm{f}}$, and successively applying the signals

$$
\mathcal{D}_{1}^{+}=\left[\begin{array}{c|c|c|c}
0 & 0 & \frac{\pi}{2} & \frac{\pi}{2} \\
\nu_{1} & \nu_{1} & -\nu_{1} & -\nu_{1} \\
\frac{\pi}{2} & \frac{\pi}{2} & 0 & 0
\end{array}\right],
$$

and $\mathcal{S}^{-}$(contracting the body link) will generate another step of rectilinear stroke with $v_{\mathrm{c}, 1}=\nu_{s} / 5$ and without triggering rigid-body tumblings. A full sequence of direct strokes has been demonstrated in Fig. 2(a). It is reminiscent of the pushme-pullyou sequence, but instead of bladders, utilizes more practical/feasible rotating circular disks. Swimming along the $x_{1}$-axis continues by repeating the 4-tuple sequence:

$$
\mathcal{S}^{+} \rightarrow \mathcal{D}_{1}^{+} \rightarrow \mathcal{S}^{-} \rightarrow \mathcal{D}_{1}^{-} .
$$

The switchings $\mathcal{S}^{+} \rightleftarrows \mathcal{S}^{-}$and $\mathcal{D}_{1}^{+} \rightleftarrows \mathcal{D}_{1}^{-}$describe delayed reciprocating actuations: there is a time delay between the termination of the body link's expansion and the beginning of its contraction. During this delay time, the disks are rotating to reach their target inclinations. Similarly, the disks remain locked when the body link is actuated.

The second swimming mode is a transverse translation normal to the plane of the I-frame as shown in Fig. 1(b). The sequence starts with initializing the configuration of four disks through the signal

$$
\mathcal{D}_{0}=\left[\begin{array}{c|c|c|c}
0 & 0 & 0 & 0 \\
-\nu_{1} & -\nu_{1} & \nu_{1} & \nu_{1} \\
-\vartheta_{0} & -\vartheta_{0} & +\vartheta_{0} & +\vartheta_{0}
\end{array}\right],
$$

and continues with the expansion of the body link by applying $\mathcal{S}^{+}$. This generates a lift with the speed

$$
v_{\mathrm{c}, 3}=\frac{\sin \left(2 \vartheta_{0}\right)}{5+\cos \left(2 \vartheta_{0}\right)} \nu_{s}
$$

in the $x_{3}$-direction. The traveling speed takes the maximum value $v_{\mathrm{c}, 3}=\nu_{s} \sqrt{6} / 12 \approx \nu_{s} / 5$ when $\cos \left(2 \vartheta_{0}\right)=$ $-1 / 5$. The second step of the transverse stroke immediately follows step 1 by terminating the expansion of the body link, actuating the disks through the control

$$
\mathcal{D}_{3}^{+}=\left[\begin{array}{c|c|c|c}
-\vartheta_{0} & -\vartheta_{0} & +\vartheta_{0} & +\vartheta_{0} \\
\nu_{1} & \nu_{1} & -\nu_{1} & -\nu_{1} \\
+\vartheta_{0} & +\vartheta_{0} & -\vartheta_{0} & -\vartheta_{0}
\end{array}\right],
$$

then contracting the body link. The resulting speed will be identical to equation (24). After the initialization step, transverse strokes persist by repeating the sequence

$$
\mathcal{S}^{+} \rightarrow \mathcal{D}_{3}^{+} \rightarrow \mathcal{S}^{-} \rightarrow \mathcal{D}_{3}^{-} .
$$

No further translational modes of swimming decoupled from rotational dynamics exist, and the Quadroar cannot perform lateral strokes in the $x_{2}$-direction because the second component of the vector $\mathbf{K}_{n} \cdot \ell_{n} \boldsymbol{e}_{1}$ is null for all $n$. Nonetheless, it can undergo yaw, pitch and roll rotations, which when followed by direct and transverse strokes give access to entire three dimensional space. Figure 3 demonstrates the steps of performing yaw and roll 
rotations about the $x_{3}$ and $x_{1}$ axes, respectively. After initialization controls that configure disks to the orientations shown in step 1 , decoupled rotations are obtained from the 4 -tuple sequences

$$
\mathcal{S}^{+} \rightarrow \mathcal{D}_{\gamma}^{+} \rightarrow \mathcal{S}^{-} \rightarrow \mathcal{D}_{\gamma}^{-}, \quad \gamma \equiv \text { yaw, roll, }
$$

where the signals $\mathcal{D}_{\gamma}^{+}$have been given in Appendix $\AA$. and corresponding angular velocities become

$$
\begin{aligned}
\text { Yaw }: \Omega_{3} & =\frac{b}{5 b^{2}+4 a^{2}+4(l+s)^{2}} \nu_{s}, \\
\text { Roll : } \Omega_{1} & =\frac{b \sin \left(2 \vartheta_{0}\right)}{4 a^{2}+b^{2}\left[5+\cos \left(2 \vartheta_{0}\right)\right]} \nu_{s}, \\
s(t) & =s_{\mathrm{i}}+\int_{0}^{t} \nu_{s}(\eta) d \eta .
\end{aligned}
$$

The maximum value of $\Omega_{1}$ corresponds to $\cos \left(2 \vartheta_{0}\right)=$ $-2 b^{2} /\left(8 a^{2}+10 b^{2}\right)$ and equals

$$
\begin{aligned}
\Omega_{1, \max } & =\frac{b \sqrt{4 a^{4}+10 a^{2} b^{2}+6 b^{4}}}{8 a^{4}+10 a^{2} b^{2}+12 b^{4}} \nu_{s}, \\
& =\frac{\sqrt{6}}{12} \frac{\nu_{s}}{b}+\mathcal{O}\left(a^{2} / b^{2}\right) .
\end{aligned}
$$

From equations (28) and (32) we conclude that with a constant value of $\nu_{s}$, roll maneuvers are performed faster than yaw ones. The pitch rotation does not require the actuation of the body link because it can be generated by the isotropic term in equation (14) if all disks rotate synchronously with the same phase angle. For $\nu_{s}=0$, we find

$$
\begin{gathered}
\Omega_{2}=-\frac{4 a^{2} \nu_{1}}{4 a^{2}+(l+s)^{2}\left[5+\cos \left(2 \vartheta_{1}\right)\right]}, \\
\vartheta_{1}=\vartheta_{2}=\vartheta_{3}=\vartheta_{4}=\int_{0}^{t} \nu_{1}(\eta) d \eta .
\end{gathered}
$$

Motion planning based on the precise control algorithms presented in this section can be readily implemented on swimmers in super-millimeter scales, where servo, piezo, or IPMC (ionic polymer metal composites) actuators and their controllers are available. In submillimeter scales, however, the body link and disks may be required to continuously driven by light or electromagnetic sources, and the only feasible input control is the frequency tuning/modulation of their reciprocating and rotational motions. Section IV explores full nonlinear, coupled roto-translatory dynamics of the Quadroar without actuation delays.

\section{CONTINUOUS ACTUATION}

We excite the body link by the harmonic signal $s=$ $\frac{1}{2} s_{0}\left[1-\cos \left(\omega_{s} t\right)\right]$ where the maximum expansion $s_{0}$ is one of our control parameters, and $\omega_{s}$ is a constant frequency. We are interested in operation modes when frequency changes in the rotational velocities of the disks,
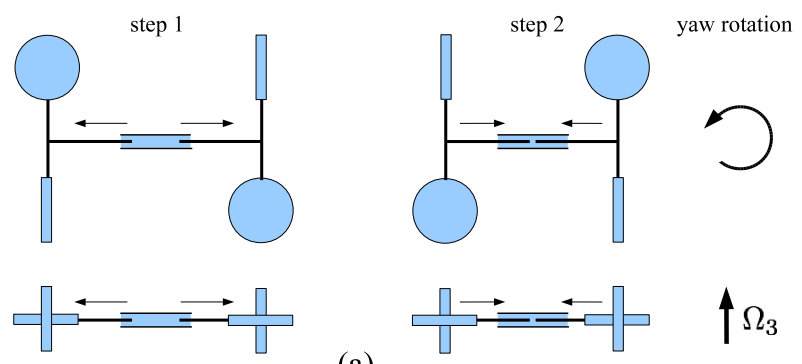

(a)
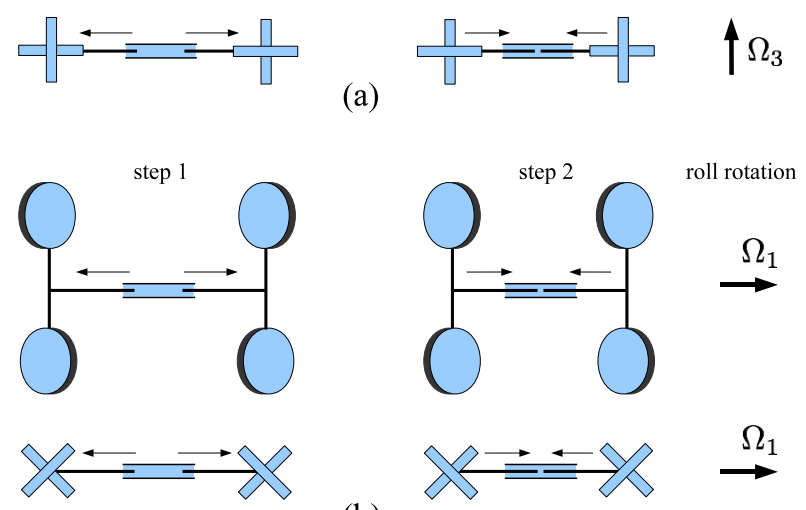

(b)

FIG. 3. Steps leading to simple yaw and roll rotations. The vectors of the resulting angular velocities have been indicated by arrows on the right hand side of step 2 . The initial alignments of the disks to their configurations shown in step 1 can be done without generating unnecessary tumblings.

and variations in the range of the expansion/contraction of the body link, can initiate maneuvers and directed motions near a stationary and non-rotating base state defined by $\omega_{s}=0$ and $\nu_{1}=\nu_{2}=-\nu_{3}=-\nu_{4}$. This study concerns disk rotation rates $\nu_{n}=(1 / k) \omega_{s}+\Delta \nu_{n}$ where $k$ is an integer number, $\Delta \nu_{n}$ is a small frequency shift, and disks are initially in phase so that $\vartheta_{n}(0)=0$ $(n=1,2,3,4)$. We refer to motions bifurcating from the explained base states as retrograde propulsion modes.

The swimmer undergoes a net directional motion along the body $x_{3}$-axis if

$$
\nu_{1}=\nu_{2}=-\nu_{3}=-\nu_{4}=\frac{1}{k} \omega_{s}, \quad k=2,4,8, \ldots,
$$

and the traveled distance increases proportional to $s_{0}$. The motion is strictly unidirectional only for $k=2$. The center of mass of the swimmer will periodically move in the transverse direction with a displacement amplitude $\sim \mathcal{O}(a)$ if $k$ be an odd integer. This simple rectilinear mode of swimming with continuous actuation is a timevarying version of the sequence shown in Fig. 2(b). For initially in phase disk rotations, substituting from $\vartheta_{0}=$ $-\omega_{s} t$ and $\nu_{s}=\dot{s}=s_{0} \omega_{s} \sin \left(\omega_{s} t\right)$ into equation (24) and integrating over $t$ gives

$$
X_{3}(t)=-s_{0} \omega_{s} \int_{0}^{t} \frac{\sin \left(\omega_{s} \eta\right) \sin \left(2 \omega_{s} \eta / k\right)}{5+\cos \left(2 \omega_{s} \eta / k\right)} d \eta .
$$

Figure 4 shows the variation of $X_{3}(t)$ for a model with $s_{0}=l / 5, b / a=l / a=5$ and for several values of $k$. The body $x_{3}$-axis is aligned with the inertial $X_{3}$-axis due to 


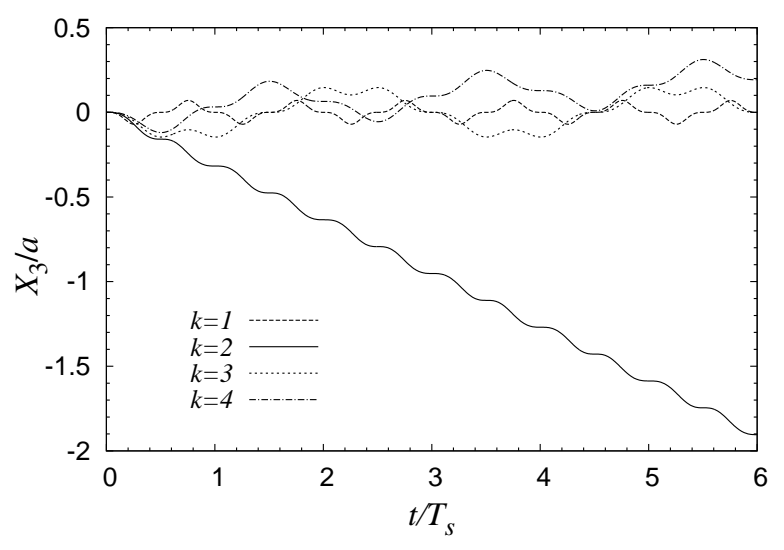

FIG. 4. Distance traveled by the swimmer in the transverse direction for a model with $s_{0}=l / 5$ and $b / a=l / a=5$. Dashed, solid, dotted and dash-dotted lines correspond to $k=$ $1,2,3,4$, respectively. Initial conditions for the position vector and orientation angles are $\left(X_{1}, X_{2}, X_{3}\right)=(0,0,0)$ and $(\phi, \theta, \psi)=(0,0,0)$. The time variable is normalized to the reciprocating period of the body link $T_{s}=2 \pi / \omega_{s}$.

our special choice of the swimmer's initial orientation. For $k=2$ one can show that

$$
\begin{aligned}
X_{3}(t)= & \frac{5 s_{0} \omega_{s} t}{2}-\frac{s_{0} \tan \left(\omega_{s} t / 2\right)}{1+\left[\tan \left(\omega_{s} t / 2\right)\right]^{2}} \\
& -2 s_{0} \sqrt{6} \arctan \left(\sqrt{6} / 3 \tan \left(\omega_{s} t / 2\right)\right) .
\end{aligned}
$$

Sampling $X_{3}(t)$ at the discrete times $t=m \pi / \omega_{s}(m=$ $1,2,3, \ldots)$ yields the recursive relation

$$
\begin{aligned}
& X_{3}(m)=X_{3}(m-1)+\left(\frac{5}{2}-\sqrt{6}\right) \pi s_{0}, \\
& X_{3}(m) \equiv X_{3}\left(t=m \pi / \omega_{s}\right) .
\end{aligned}
$$

Therefore, the longest step that the Quadroar can take in the retrograde propulsive mode over a cycle of the body link's actuation is $(5-2 \sqrt{6}) \pi s_{0} \approx 0.317 s_{0}$.

Interesting motions occur when the rotational speeds of the disks are detuned, and the swimmer tumbles as it moves in the three dimensional space. The simplest roto-translatory mode of swimming is when the motion is confined to a plane, and only the pitch angle $\theta$ varies over time. Figure 5 shows the Quadroar's trajectory for $b / a=l / a=5, s_{0}=2 l / 3$, and rotational frequencies $\nu_{1}=\nu_{2}=\omega_{s} / 2$ and $\nu_{3}=\nu_{4}=-\omega_{s} /(2+\epsilon)$. The orbit corresponding to $\epsilon=0.007$ is an almost periodic rosette with 7 lobes and triple hierarchical loops at the turning points (Fig. 5(a)). The innermost loop is a cardioid. By decreasing $\epsilon$ to 0.004 we obtain a quasi-periodic orbit containing 4 centrophillic multiloops on its periphery (Fig. 5(b)). Each multiloop bundle now consists of 6 loops. The motion on quasi-periodic orbits that we find exhibit two generic phases: lingering while the swimmer undergoes a cardioid or multiloop turn, followed by a propulsive phase of long-distance travel. The mean radii of orbits, the number of loops in lingering phases and the centrophillic or centrophobic alignments of the multiloop bundles nonlinearly depend on $s_{0}$ and $\epsilon$. For instance, setting $\epsilon=0.0051$ yields a slowly precessing orbit with 10 centrophobic multiloop bundles (Fig. 5(c)).

We conduct further survey in the parameter space by keeping $\epsilon$ fixed and varying $s_{0}$. Figure 6 shows that the mean diameter of the quasi-periodic trajectory is boosted significantly by taking $s_{0}=l / 2$ and $\epsilon=0.005$, but it drops by decreasing $s_{0}$. The pattern demonstrated in Fig. 6(c) has the least number of loops at the periphery of the orbit. Its turning loop is a single cardioid. Figure 7 displays a single cardioid and a multiloop structure. The existence of single or multiple loop bundles together with long-distance swims can be explained in the context of resonances. Since the front and rear disks rotate with different angular velocities, even though the frequency differences are small, phase shifts occur over time. The Quadroar propels on an almost straight line when the phase angles are such that

$$
\vartheta_{1} \approx-\vartheta_{3}, \quad \cos \left(2 \vartheta_{1}\right) \approx-1 / 5,
$$

and the disks are in the relative configurations of Fig. 2(b) needed for the transverse propulsion mode. It is remarked that $\vartheta_{2}=\vartheta_{1}$ and $\vartheta_{4}=\vartheta_{3}$ for the planar swimming modes of Figs 5 and 6 The swimmer moves on a multiloop pattern when all $\vartheta_{n}$ have the same sign. Motions on curved paths will thus be intermediary phases with $\operatorname{sign}\left(\vartheta_{1}\right)=-\operatorname{sign}\left(\vartheta_{3}\right)$.

Our numerical simulations show that the rotational speeds $\nu_{1}=-\nu_{3}=\omega_{s} / k$ and $\nu_{2}=-\nu_{4}=\omega_{s} /(k+\epsilon)$ induce generally quasi-periodic motions in the frontal $\left(x_{2}, x_{3}\right)$-plane of the swimmer. Similar to motions in the $\left(x_{1}, x_{3}\right)$-plane, the mean radii of orbits can be orders of magnitudes larger than the size of the swimmer. A full three dimensional motion occurs when no mirror and central symmetries exist in the magnitudes of $\nu_{n}$, i.e., $\nu_{1} \neq \nu_{2}$ and $\left|\nu_{1}\right| \neq\left|\nu_{3}\right|$. This extends the number of control parameters to 5 , and we need to tune four frequency ratios/shifts along with $s_{0}$ to generate desired bounded orbits. The most interesting combination of disk rotation rates is $\nu_{1}=\omega_{s} / 2, \nu_{2}=\omega_{s} /(2+\epsilon), \nu_{3}=-\omega_{s} / 2$ and $\nu_{4}=-\omega_{s} /(2-\epsilon)$, which together with the stroke length $s_{0}=l / 2$ yield the three dimensional quasi-periodic orbit of Fig. 8. It is seen that the orbit has three screw-shaped lobes whose projections on the $\left(X_{1}, X_{3}\right)$ and $\left(X_{2}, X_{3}\right)$ coordinate planes precess. The orbit makes an inclined structure in the $\left(X_{1}, X_{2}\right)$-plane. The screw-shape trajectory is due to a combination of roll and yaw rotations and the translation along the body $x_{3}$-axis. In this operation mode the swimmer also performs pitch rotations when the phase angles of all disks have the same sign. The inclination of the resulting orbit in the inertial frame depends on the initial orientation of the swimmer that can be adjusted by simple maneuvers introduced in section III. Further intentional shifts in the frequencies of the disks can also put the swimmer on transitional paths and change its attitude. We note that the spatial orientation 

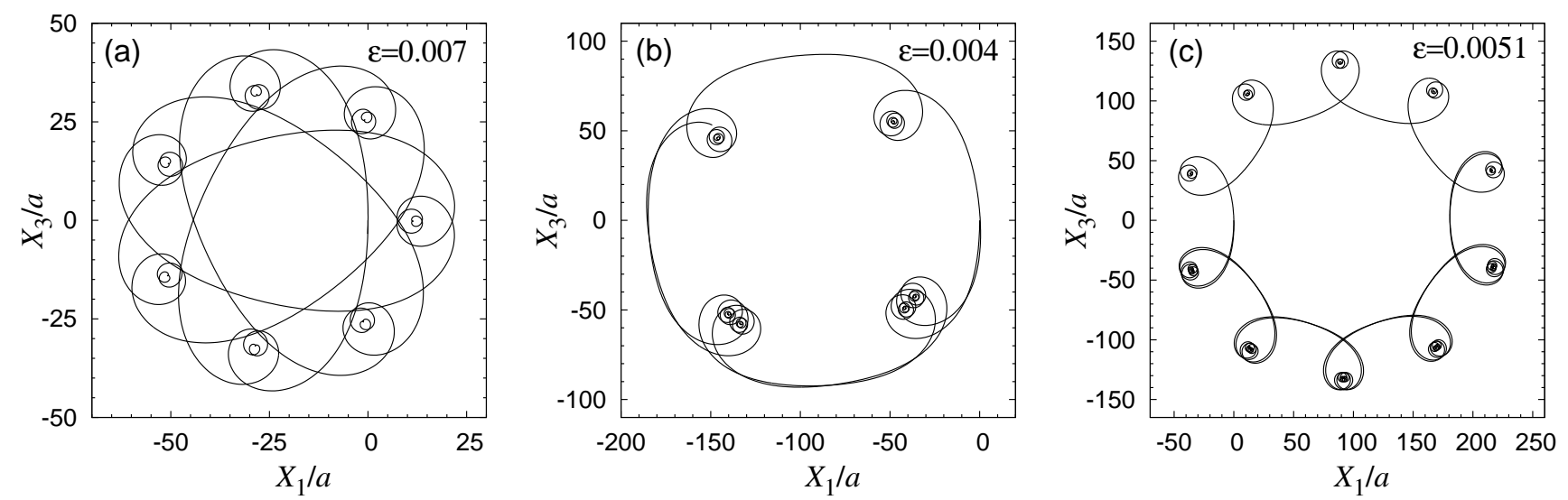

FIG. 5. Trajectories of the swimmer in the retrograde propulsion mode. Geometrical parameters have been set to $b / a=l / a=5$, the maximum stroke of the body link is $s_{0}=2 l / 3$, and the rotational velocities of the disks are $\nu_{1}=\nu_{2}=\omega_{s} / 2$ and $\nu_{3}=\nu_{4}=-\omega_{s} /(2+\epsilon)$. Initial conditions for the position vector and orientation angles are $\left(X_{1}, X_{2}, X_{3}\right)=(0,0,0)$ and $(\phi, \theta, \psi)=(0,0,0)$, respectively. Note the different scales of the panels. The maximum integration times $\left(t_{\max }\right)$ of orbits are also different. (a) An almost periodic rosette orbit with $\epsilon=0.007$ and $t_{\max }=2010 T_{s}$ where $T_{s}=2 \pi / \omega_{s}$. (b) A quasi-periodic orbit with 4 multiloop bundles and $\epsilon=0.004$ and $t_{\max }=3100 T_{s}$. (c) A slowly precessing quasi-periodic orbit with $\epsilon=0.0051$ and $t_{\max }=6000 T_{s}$.
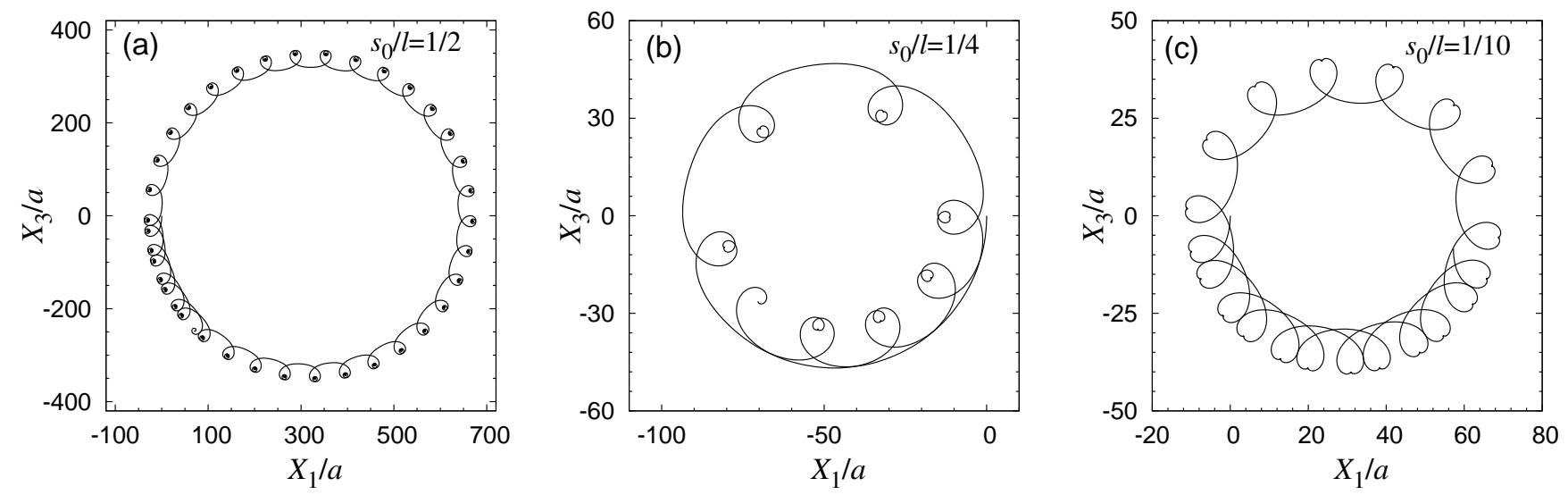

FIG. 6. Same as Fig. 5 but for models with $\epsilon=0.005$ and three choices of $s_{0} / l$. All orbits are quasi-periodic. (a) $s_{0} / l=1 / 2$ and $t_{\max }=15000 T_{s}$. (b) $s_{0} / l=1 / 4$ and $t_{\max }=3000 T_{s}$. (c) $s_{0} / l=1 / 10$ and $t_{\max }=8000 T_{s}$.

of orbits with initially non-zero and unequal phase angles are not necessarily identical to the ones with initially zero phases that we experimented here.

\section{DISCUSSIONS}

Swimming in more than one dimensions involves the attitude and angular velocity calculations of the swimmer. The set of four disks together with the thin and frictionless I-frame result in simple translation and resistance matrices, which were used to generate basic displacements and attitude maneuvers through sending delayed pulses to the actuators. Since spins and translations can be performed independently, we have basically a zero-turning-radius swimmer. These two properties of- fer a wide range of capabilities for non-contact fluidic manipulation [18].

Coupled roto-translatory motions with the continuous operations of the actuators led to complex yet geometrically well-defined orbital patterns, which were found to be sensitive to the stroke length of the body link. Our study is restricted to rotational frequencies of disks that satisfy $\nu_{n}=\omega_{s} / k+\Delta \nu_{n}$, and for operation modes that require disks to start their rotation in phase. Even with these limited sets of model parameters, the Quadroar showed to have a rich dynamics. In continuous retrograde propulsion modes it moves on periodic and quasi-periodic orbits with mean diameters orders of magnitude larger than its body size, and its trajectory becomes dense in the invariant measure of quasi-periodic orbits. This exceptional capability makes it a candidate for accurate 

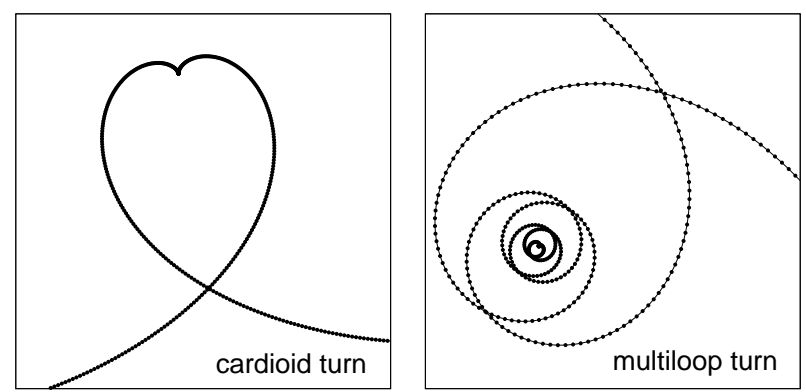

FIG. 7. Cardioid (left) and multiloop (right) turning paths. In both cases the trajectory becomes cuspy and nondifferentiable at one position. The innermost loop of the multiloop bundle is a cardioid.
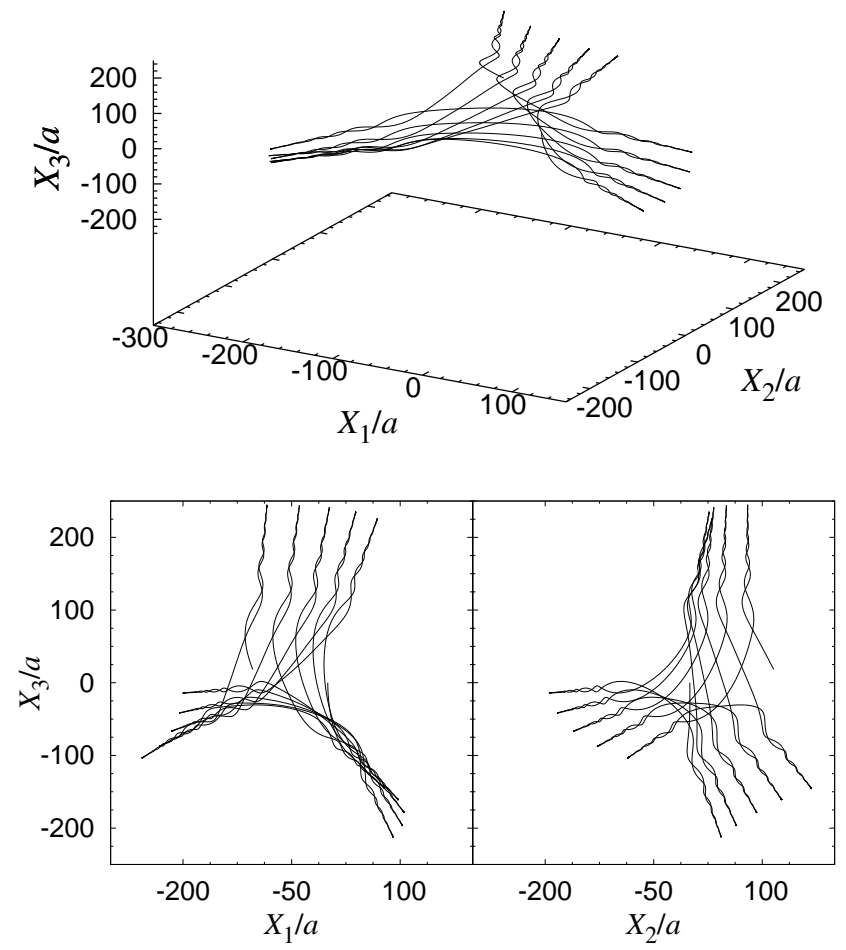

FIG. 8. A three dimensional quasi-periodic orbit and its projections on the $\left(X_{1}, X_{3}\right)$ and $\left(X_{2}, X_{3}\right)$ coordinate planes for a model with $b / a=l / a=5, s_{0}=l / 2, \nu_{1}=\omega_{s} / 2$, $\nu_{2}=\omega_{s} /(2+\epsilon), \nu_{3}=-\omega_{s} / 2$ and $\nu_{4}=-\omega_{s} /(2-\epsilon)$ where $\epsilon=0.002$. The integration time is $t_{\max }=15000 T_{s}$ and the swimmer starts its motion from $\left(X_{1}, X_{2}, X_{3}\right)=(0,0,0)$. This is a swimming mode with retrograde propulsion and has screw-shape lobes with cusped turning points. The projections of the lobes precess in the coordinate planes.

search and scanning purposes in low Reynolds number regimes. It is worth noting that quasi-periodic orbits have also been observed in the motion of planetary and stellar systems [19]. Both planar and three dimensional quasi-periodic orbits involved slow turning motions on non-differentiable curves, as shown in Fig. 7 A full understanding of the correlation between the topology of orbits and the control parameters $s_{0}$ and $\nu_{n} / \omega_{s}$, and the existence of chaotic movements are interesting problems for future work.

Swimmers induce a flow field that contributes to the mixing of the surrounding fluid. Such a mixing in low Reynolds number regimes benefits from the long-range nature of the velocity field created by the swimmer, but suffers from the lack of turbulence. Mixing in low Reynolds number is important as it enhances the rate of the particle diffusion in the fluid which results in, for example, a more efficient nutrient supply to microorganisms [20]. It is, in fact, believed that the rational behind the motion of microorganisms, besides immediate survival, is partly to optimally distribute the nutrients [21].

Low Reynolds number swimmers are known to enhance mixing. Over the surface of a soap film it has been observed that bacteria induce super-diffusion to the nearby fluid particles [21]. Motile bacteria also observed to enhance the mixing at the interface of two streams of fluid 22, 23], and a flat rigid surface covered with restrained bacteria can significantly increase the mixing of adjacent fluid particle 24]. While the details of flow pattern produced by the Quadroar is beyond the scope of this article and deserves an independent investigation, we would like to comment that the broad family of rich sweeping patterns, that can be periodic, quasi-periodic and potentially chaotic, can result in a wide range of complex flow fields capable of driving an efficient mixing, which is currently achieved by boundary actuation [25] or externally actuated stirring [26]. Rotating disks of the Quadroar can be considered as micro-impellers [27, 28] that not only directly stir the flow, but also propel the Quadroar forward along specific yet complex trajectories to achieve maximum efficiency stirring. If stirring is the only objective, disks may be further modified but this will clearly also affect the overall trajectory of the Quadroar, and therefore a careful optimization must be carried out beforehand.

With current developments in nano sciences and technology, the Quadroar seems to be a realizable swimmer in molecular scales. For more than a decade, several groups of organic chemists have been designing and synthesizing molecular motors that work by electrochemical, photochemical or thermal actuations [29, 30]. One of the prominent examples in this line, is the unidirectional light-driven motor of Koumura et al. 31 based on symmetric biphenanthrylidenes, and its second generation [32] that can be connected to a supporting frame. The unidirectional motion of this nano motor is maintained by a stereogenic center, and the helix inversion of the molecule through irradiation or heating. These motors can be used as the driving units of various complexes, including nano cars with carborane wheels [33]. A recently experimented electrically driven wheeled molecule uses four unidirectional rotary motors to move on metal surfaces. A similar concept can be used to synthesize a nano-scale Quadroar, but we also need a light-driven molecular actuator that functions like the telescopic body link of our swimmer. This central unit can be designed in 
a number of ways: (i) using an azobenzene chassis that expands and contracts through cis-trans photoisomerization. (ii) adapting a mechano-sensitive channel protein that can open and close by successive irradiation of ultraviolet and visible lights [34]. Azobenzene chromophores have been successfully applied in synthesizing photoactive nanoworms [35] and molecular scissors [36], and seem to be more feasible units for generating the open-close function of the body link of a nano-scale Quadroar.

\section{Appendix A: Rotation and Transfer Matrices}

The transformation matrices for roll-pitch-yaw rotations are defined as

$$
\begin{aligned}
& \text { Yaw : } \mathbf{R}_{x_{3}}(\psi)=\left[\begin{array}{ccc}
\cos \psi & \sin \psi & 0 \\
-\sin \psi & \cos \psi & 0 \\
0 & 0 & 1
\end{array}\right], \\
& \text { Pitch : } \mathbf{R}_{x_{2}}(\theta)=\left[\begin{array}{ccc}
\cos \theta & 0 & -\sin \theta \\
0 & 1 & 0 \\
\sin \theta & 0 & \cos \theta
\end{array}\right] \text {, } \\
& \text { Roll : } \mathbf{R}_{x_{1}}(\phi)=\left[\begin{array}{ccc}
1 & 0 & 0 \\
0 & \cos \phi & \sin \phi \\
0 & -\sin \phi & \cos \phi
\end{array}\right] .
\end{aligned}
$$

The elements of the resistance matrices $\mathbf{C}_{\alpha \beta}=C_{\alpha \beta, i j} \boldsymbol{e}_{i} \boldsymbol{e}_{j}$ and the components of the forcing vectors $\boldsymbol{f}_{\alpha}=f_{\alpha, i} \boldsymbol{e}_{i}$ $(\alpha, \beta=1,2)$ are:

$$
\begin{gathered}
\mathbf{C}_{11}=\sum_{n=1}^{4} \mathbf{K}_{n} \cdot \mathbf{R}, \\
C_{12, i j}=\sum_{n=1}^{4} K_{n, i k} r_{n, m} \varepsilon_{m k j}, \\
C_{21, i j}=\sum_{n=1}^{4} \varepsilon_{i m k} r_{n, m} K_{n, k l} R_{l j}, \\
C_{22, i j}=\sum_{n=1}^{4}\left(\frac{32 a^{3}}{3} \delta_{i j}+\varepsilon_{i m k} \varepsilon_{l j p} r_{n, m} r_{n, p} K_{n, k l}\right),
\end{gathered}
$$

[1] E. M. Purcell, Am. J. Phys. 45, 3 (1977).

[2] E. Lauga, and T. R. Powers, Rep. Prog. Phys. 72, 096601 (2009).

[3] A. Najafi and R. Golestanian, Phys. Rev. E 69, 062901 (2004).

[4] R. Golestanian and A. Ajdari, Phys. Rev. E 77, 036308 (2008).

$$
\begin{gathered}
f_{1, i}=-\sum_{n=1}^{4} K_{n, i 1} \dot{\ell}_{n}, \\
f_{2, i}=-\sum_{n=1}^{4}\left(\frac{32 a^{3}}{3} \delta_{2 i} \dot{\vartheta}_{n}+\dot{\ell}_{n} r_{n, j} K_{n, k 1} \varepsilon_{i j k}\right),
\end{gathered}
$$

where $\delta_{i j}$ is Kronecker's delta and $\varepsilon_{i j k}$ is the permutation symbol. With reciprocating actuators, periodic control signals for the yaw and roll rotations are computed from

$$
\begin{gathered}
\mathcal{D}_{\text {yaw }}^{+}=\left[\begin{array}{c|c|c|c}
-\frac{\pi}{2} & 0 & 0 & +\frac{\pi}{2} \\
\nu_{1} & \nu_{1} & -\nu_{1} & -\nu_{1} \\
0 & \frac{\pi}{2} & -\frac{\pi}{2} & 0
\end{array}\right], \\
\mathcal{D}_{\text {roll }}^{+}=\left[\begin{array}{c|c|c|c}
-\vartheta_{0} & +\vartheta_{0} & +\vartheta_{0} & -\vartheta_{0} \\
\nu_{1} & -\nu_{1} & -\nu_{1} & \nu_{1} \\
+\vartheta_{0} & -\vartheta_{0} & -\vartheta_{0} & +\vartheta_{0}
\end{array}\right] .
\end{gathered}
$$

\section{Appendix B: Unit Quaternions}

While integrating the equations $\dot{\alpha}=\mathbf{T}^{-1} \cdot \boldsymbol{\Omega}$ to determine the orientation of the swimmer, a geometrical singularity occurs at $\theta= \pm \pi / 2$. One way of resolving such singularities is to switch between roll-pitch-yaw and other sets of Euler angles. Alternative approach, which we adopt in this study, is to express the attitude dynamics in terms of the unit quaternions:

$$
\boldsymbol{q}=\left(\begin{array}{c}
q_{0} \\
q_{1} \\
q_{2} \\
q_{3}
\end{array}\right)=\left(\begin{array}{c}
c_{\frac{\phi}{2}} c_{\frac{\theta}{2}} c_{\frac{\psi}{2}}+s_{\frac{\phi}{2}} s_{\frac{\theta}{2}} s_{\frac{\psi}{2}} \\
-c_{\frac{\phi}{2}} s_{\frac{\theta}{2}} s_{\frac{\psi}{2}}+s_{\frac{\phi}{2}} c_{\frac{\theta}{2}} c_{\frac{\psi}{2}} \\
c_{\frac{\phi}{2}} s_{\frac{\theta}{2}} c_{\frac{\psi}{2}}+s_{\frac{\phi}{2}} c_{\frac{\theta}{2}} s_{\frac{\psi}{2}} \\
c_{\frac{\phi}{2}} c_{\frac{\theta}{2}} s_{\frac{\psi}{2}}-s_{\frac{\phi}{2}} s_{\frac{\theta}{2}} c_{\frac{\psi}{2}}
\end{array}\right)
$$

whose dynamics is governed by

$$
\dot{\boldsymbol{q}}=\frac{1}{2}\left[\begin{array}{cccc}
0 & -\Omega_{1} & -\Omega_{2} & -\Omega_{3} \\
\Omega_{1} & 0 & \Omega_{3} & -\Omega_{2} \\
\Omega_{2} & -\Omega_{3} & 0 & \Omega_{1} \\
\Omega_{3} & \Omega_{2} & -\Omega_{1} & 0
\end{array}\right] \cdot \boldsymbol{q}
$$

After integrating these equations in the time domain, inverse transformation to the space of roll-pitch-yaw angle is carried out using the following relations

$$
\boldsymbol{\alpha}=\left(\begin{array}{c}
\operatorname{atan} 2\left(2 q_{2} q_{3}+2 q_{0} q_{1}, q_{3}^{2}-q_{2}^{2}-q_{1}^{2}+q_{0}^{2}\right) \\
-\arcsin \left(2 q_{1} q_{3}-2 q_{0} q_{2}\right) \\
\operatorname{atan} 2\left(2 q_{1} q_{2}+2 q_{0} q_{3}, q_{1}^{2}+q_{0}^{2}-q_{3}^{2}-q_{2}^{2}\right)
\end{array}\right)
$$

[5] G. P. Alexander, C. M. Pooley, and J. M. Yeomans, J. Phys.: Condens. Matter 21, 204108 (2009).

[6] F. Alouges, A. DeSimone, and A. Lefebvre, J. Nonlinear Sci. 18, 277 (2008).

[7] M. Leoni, J. Kotar, B. Bassetti, P. Cicuta, and M. C. Lagomarsino, Soft Matter 5, 472 (2009). 
[8] R. Dreyfus, J. Baudry, and H. A. Stone, Eur. Phys. J. B 47, 161 (2005).

[9] J. E. Avron, O. Kenneth, and D. H. Oaknin, New Journal of Physics 7, 234 (2005).

[10] R. Ledesma-Aguilar, H. Löwen, and J. M. Yeomans, Eur. Phys. J. E 35, 70 (2012).

[11] F. Alouges, A. DeSimone, L. Heltai, A. Lefebvre-Lepot, and B. Merlet, arXiv:1007.4920v2 (2011).

[12] A. Najafi and R. Zargar, Phys. Rev. E 81, 067301 (2010).

[13] A. Shapere and F. Wilczek, J. Fluid Mech. 198, 557 (1989).

[14] J. E. Avron, O. Gat, and O. Kenneth, Phys. Rev. Lett. 93, 186001 (2004).

[15] E. Setter, I. Bucher, and S. Haber, Phys. Rev. E 85, 066304 (2012).

[16] F. Alouges, A. DeSimone, L. Giraldi, and M. Zoppello, International Journal of Non-Linear Mechanics 56, 132 (2013).

[17] J. Happel, and H. Brenner, Low Reynolds Number Hydrodynamics (Martinus Nijhoff Publishers, The Hague, 1983).

[18] S. Floyd, C. Pawashe, and M. Sitti, IEEE Transactions on Robotics 25, 1332 (2009).

[19] J. Binney, and S. Tremaine, Galactic Dynamics, 2nd edition, (Princeton University Press, Princeton, 2008)

[20] D. O. Pushkin, and J. M. Yeomans, Phys. Rev. Lett. 111, 188101 (2013).

[21] G. Grégoire, H. Chaté, and Y. Tu, Phys. Rev. Lett. 86, 556 (2001).

[22] M. J. Kim, and K. S. Breuer, Physics of Fluids 16, L78 (2004).
[23] M. J. Kim, and K. S. Breuer, Journal of Fluids Engineering 129, 319 (2007).

[24] N. Darnton, L. Turner, K. Breuer, and H. C. Berg, Biophysical journal 86, 1863 (2004).

[25] G. Mathew, I. Mezić, S. Grivopoulos, U. Vaidya, and L. Petzold, J. Fluid Mech. 580, 261 (2007).

[26] I. J. Couchman, and E. C. Kerrigan, Journal of Process Control 20, 1103 (2010).

[27] L. Yang, J. Cheng, P. Fan, C. Yang, and Z. Mao, Chemical Engineering and Technology 36, 443 (2013).

[28] V. Hessel, H. L'owe, and F. Sch'onfeld, Chemical Engineering Science 60, 2479 (2005).

[29] G. S. Kottas, L. I. Clarke, D. Horinek, and J. Michl, Chem. Rev. 105, 1281 (2005)

[30] J. Michl, and E. C. H. Sykes, ACS Nano 3, 1042 (2009)

[31] N. Koumura, R. W. J. Zijlstra, R. A. van Delden, N. Harada, and B. L. Feringa, Nature 401, 152 (1999)

[32] N. Koumura, E. M. Geertsema, M. B. van Gelder, A. Meetsma, and B. L. Feringa, J. Am. Chem. Soc. 124, 5037 (2002)

[33] P.-T. Chiang, J. Mielke, J. Godoy, J. M. Guerrero, L. B. Alemany, C. J. Villagómez, A. Saywell, L. Grill, and J. M. Tour, ACS Nano 6, 592 (2012)

[34] A. Koçer, M. Walko, W. Meijberg, and B. L. Feringa, Science 309, 755 (2005)

[35] T. Sasaki, and J. M. Tour, Organic Letters 10, 897 (2008)

[36] T. Muraoka, K. Kinbara, Y. Kobayashi, and T. Aida, J. Am. Chem. Soc. 125, 5612 (2003) 